\title{
多线摇摆往复式线锯切割加工运动的理论 及试验研究
}

\author{
杨 沁 黄 辉 郑生龙 \\ (华侨大学制造工程研究院 厦门 361021)
}

\begin{abstract}
摘要: 多线摇摆往复式切割加工因其切割效率高和加工精度好的优点, 现已逐渐成为硬脆材料晶圆的主要切割方式; 分析了 多线摇摆往复式切割加工中三个主要的运动方式, 建立了多线摇摆往复式切割加工运动的理论模型, 推导了线锯摇摆切割过 程中的材料去除率 $(Q)$ 和单位长度新线材料去除体积 $(R)$ 的理论计算公式; 理论分析了加工工艺参数对 $Q$ 和 $R$ 的影响规律; 从 加工运动学的角度提出了固定 $R$ 的线锯切割新构想, 并逆向推导了晶圆切割过程的相关工艺参数变化曲线; 通过与工厂现有 加工工艺的线锯切割试验比较，证实本文所提出的线锯切割新构想能有效地提高晶圆切割质量。
\end{abstract}

关键词: 多线摇摆往复切割; 材料去除率; 单位长度新线材料去除体积; 切割质量

中图分类号: TG6

\section{Theoretical and Experimental Research on Machining Movement of Multi-wire Sawing with Rocking and Reciprocating}

\author{
YANG Qin HUANG Hui ZHENG Shenglong
}

(Institute of Manufacturing Engineering, Huaqiao University, Xiamen 361021)

\begin{abstract}
Multi-wire sawing with rocking and reciprocating has gradually become a main way to slice the hard and brittle material wafers due to its high efficiency and good accuracy. Three main motions in multi-wire sawing with rocking and reciprocating are analyzed. The theoretical models of three main motions are established. The theoretical derivation of the material removal rate $(Q)$ and the material removal volume of new wire with per unit length $(R)$ in the sawing process is proposed. The influence of processing parameters on $Q$ and $R$ is analyzed theoretically. A new concept of wire sawing with fixing $R$ is proposed according the kinematics. The relevant process parameters variation curve in the wafer sawing process is derived inversely. It is found that the new sawing method improved the wafer sawing quality effectively compared with the actual sawing experimental results of the factory.
\end{abstract}

Key words: multi-wire sawing with rocking and reciprocating; material removal rate; material removal volume of new wire with per unit length; sawing quality

\section{0 前言}

硬脆晶体材料如硅、蓝宝石、碳化硅等广泛应 用于纯电动汽车、太阳能光伏、风力发电、高铁及 轨道交通、智能电网、智能家电以及航空航天等产 业领域 ${ }^{[1]}$ 。硬脆晶体材料从长晶到应用生产需要经 过多个生产工序，其上游生产的主要加工环节为:

* 国家自然科学基金(51375179)、福建省高校产学研项目(2017H6014)、 教育部创新团队 (IRT_17R41)和华侨大学研究生科研创新基金 (17013080041)资助项目。20191011 收到初稿, 20191220 收到 修改稿
切割-研磨-抛光 ${ }^{[2]}$ 。切割是晶棒加工的第一道工 序, 其目的是将晶棒切成厚度不足 $1 \mathrm{~mm}$ 的片状, 其切割质量对后续的研磨抛光有着重要的影响。

目前晶体材料常用的切割加工主要是利用线锯切 割完成。

线锯切割加工根据切片数量, 可以分为单线切 割和多线切割。单线切割每次只能完成一片晶圆的 加工。对于单线切割, 学者们利用压痕断裂力学的 方法研究了线锯切割过程中的材料去除机理 ${ }^{[3]}$ 。同 时研究了线锯运动速度、进给速度、线锯直径以及 线锯张紧力等工艺参数对切割力及切割表面质量的 影响 ${ }^{[4-7]}$ 。切割片的表面质量主要通过晶片厚度、表 
面形貌、表面粗粘度和亚表面裂纹等几个方面进行

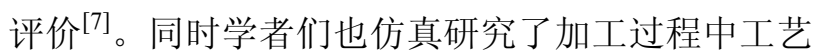
参数对切削温度以及损伤层厚度的影响规律 ${ }^{[8-10]}$ 。 也有学者提出了新型线锯切割技术以提高线锯切割 质量 ${ }^{[11]}$ 。

由于单线切割加工效率较低, 在实际生产中则 主要是利用多线切割加工的方式。多线切割与单线 切割相比, 每次可以完成数百片晶圆的加工, 其加 工效率明显提高。对于游离磨料多线切割, 学者们 从断裂力学和压痕试验的角度研究了材料微观锯切 机理, 并建立起了游离磨粒多线切割的理论模 型 ${ }^{[12]}$ 。对于固结磨料多线切割, 学者们分析切割过 程中线速度、进给速度、张紧力、切割长度对锯切 力以及线弓的影响, 建立了线锯宏观切割模型 ${ }^{[13]}$; 分析了耗线量和线锯初始弯曲量对切割力的影 响 ${ }^{[14-15]}$ 。学者们还从仿真和试验两个方面研究了多 线切割过程中的温度场变化 ${ }^{[16-18]}$ 。对于多线切割的 加工质量研究则主要集中在分析工艺参数对晶片面 型精度的影响 ${ }^{[19]}$; 也有学者针对多线往复式线锯切 割的运动过程进行了分析, 建立运动理论模型, 提 出材料去除率的理论模型, 通过切割试验验证其影 响规律 ${ }^{[20]}$ 。以上学者的研究成果对推动多线切割加 工的应用提供了许多理论依据和实际指导意义。

虽然多线切割加工有效地提高了切割效率, 但 受到晶圆形状的影响, 导致线与晶圆的接触长度随 着切割过程有很大的变化, 从而对加工质量产生影 响。为了减小切割过程中线与晶圆接触长度的变化, 线锯切割设备生产厂商提出了在切割过程中加入摇 摆运动。HARDIN 和 CLARK 分别在单线切割机上 研究了摇摆角度和摇摆频率对锯切力、线弓角和锯 切表面粗糙度的影响 ${ }^{[6,21]}$ 。其研究成果表明摇摆运 动具有减少线材磨损和提高切割质量等优点。然而 对于多线摇摆往复线锯切割而言, 随着摇摆运动的 加入, 线锯切割的运动方式更为复杂, 可调加工工 艺参数增加, 并且工艺参数之间也会相互影响, 从 而导致实际加工质量的可控性变差。以往以单因素 法来分析工艺参数对加工质量的影响难以得到较好 的应用。

本文针对多线摇摆往复式线锯切割的加工运动 方式进行了理论建模; 推导了晶圆切割过程中材料 去除率 $(Q)$ 和单位长度新线材料去除体积 $(R)$ 的理论 计算公式, 分析了工件进给运动、工件摇摆运动以 及线锯往复运动对 $Q$ 和 $R$ 的影响规律; 在此基础上 针对晶圆形状的影响, 提出了基于固定 $R$ 的线锯切 割加工的新思路, 逆向推导了保证固定 $R$ 加工时的
工件进给速度和新线进给速度的理论公式; 并通过 多线摇摆切割试验对所提出固定 $R$ 加工的新思路进 行了试验验证。

\section{1 多线摇摆往复切割加工运动的理论 模型}

虽然不同公司的设备略有不同，但多线摇摆往 复式切割的基本运动方式如图 1 所示, 可分解为三 个相对独立的运动方式: 线锯的往复运动、工件的 进给运动以及工件的摇摆运动。

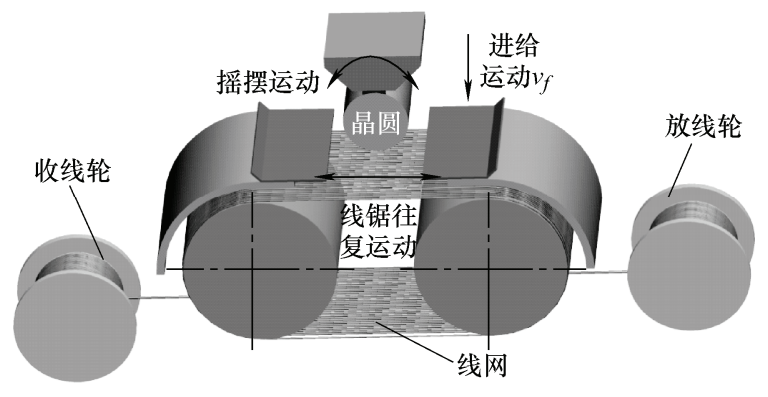

图 1 多线摇摆往复切割运动示意图

\section{1 线锯往复运动}

加工过程中, 线锯的往复运动是通过左右导轮 的周期性正反转带动来完成的。其目的是保证线锯 有一定的切割速度以及切割过程中持续提供新线。

图 2 是线锯一个往复运动中速度随时间的变化曲 线。图中 $v_{s}$ 是线锯在稳定切割时的速度, 也是往复 运动中能达到的最大速度, $\boldsymbol{v}_{s}$ 表示线锯反向运动, $t_{f}, t_{b}$ 分别是线锯正向匀速运动以及反向匀速运动的 时间, $t_{a}$ 为线锯从速度为 0 加速到最大速度时所用 的时间, $t_{d}$ 为线锯从最大速度减少到 0 时所用的时 间。一般情况下，往复运动中线锯的加速度保持不 变。在实际切割中为了保证线网的锯切能力, 一般 导轮正转的时间 $t_{f}$ 大于反转的时间 $t_{b}$, 此时线锯向 前运动的长度将大于反向运动的长度, 一部分线锯 将被收线轮收集不再参与切割, 而在下一个往复运 动中又有部分的新线从放线轮放出参与切割。如此 往复运动实现切割过程中新线不断的供给, 旧线不 断的回收, 使线网上的线不断地得到更新, 保证了 线网的锯切能力。

用新线进给速度 $v_{w}$ 来表征单位时间内进入锯 切加工弧区中的新线长度; 在完成晶圆切割总时间 $T$ 内, 切割所用的总耗线量 $M$ 可由不同切割位置处 的新线进给速度通过式(1)计算所得

$$
M=\int_{0}^{T} v_{w}(y) \mathrm{d} t
$$




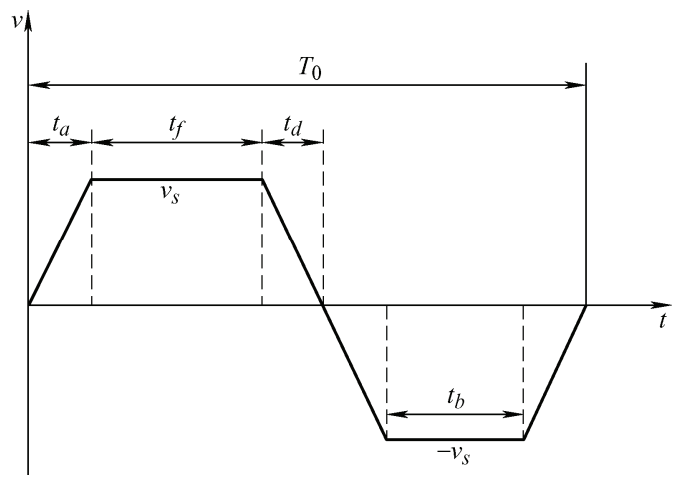

图 2 线网往返周期运动

\section{2 工件进给运动}

工件进给运动是保证切割过程的连续进行, 完 成整个晶圆面的切割, $v_{f}$ 为工件沿 $y$ 方向不同位置 的进给速度, 如图 3 所示。

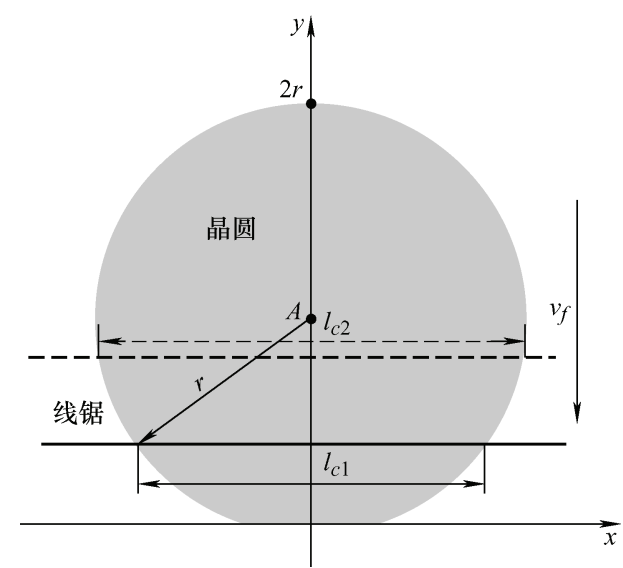

图 3 线锯接触长度随切割位置 $y$ 的变化示意图

由于晶圆为圆形, 线锯与工件的接触长度 $l_{c}$ 随 加工过程而产生变化, 见图 3 所示。实际生产中, 常通过调整不同位置处的工件进给速度来适应于线 锯接触长度的变化, 以保证线锯切割过程中的稳定 性。为了不影响整片的切割总时间。通过控制工件 中间位置的进给速度与工件切出位置的进给速度的 比值(进给速比 $u$ ) 来对不同位置的切割速度进行分 配。进给速比 $u$ 如式(2)所示 ${ }^{[20]}$ 。

$$
u=\frac{v_{f m}}{v_{f 0}}
$$

式中, $v_{f m}$ 为 $y=r$ 时的进给速度, $v_{f 0}$ 为 $y=0$, 或者 $y=2 r$ 时的进给速度。文献[20]中在无摇摆条件下, 根据 接触长度的变化对进给速度进行调节, 得到进给速 度随切割位置 $y$ 的表达式

$$
v_{f}(y)=\frac{2 r^{3}(2+u)}{3 T\left[r^{2}-(r-y)^{2}(1-u)\right]}
$$

\section{3 工件摇摆运动}

加工过程中, 工件摇摆运动的简化示意图如 图 4 所示, 工件装夹在固于弧形导轨的摇摆块上,
并在步进电动机的控制下进行周期性左右摇摆运 动。工件摇摆的左右极限位置与摇摆中心线的夹角 称为最大摇摆角度; 图 4 中虚线表示晶片处于中间 位置，而黑色实线表示晶圆沿着圆心摇摆到最大角 度 $\theta$ 位置处。

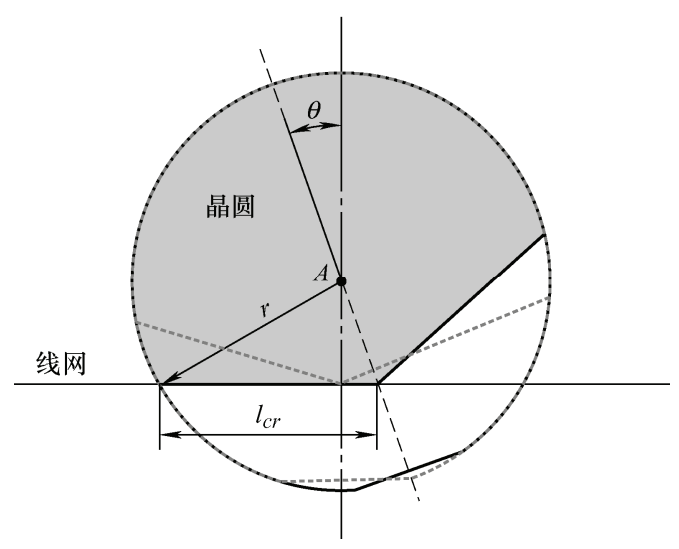

图 4 工件摇摆示意图

\section{2 多线摇摆往复切割的材料去除率}

\section{1 多线摇摆往复切割的材料去除率模型}

线锯切割过程中材料去除率 $Q$ 可由式(4)算得。

$$
Q=v_{f} l_{c} d
$$

式中, $d$ 为锯缝宽度, 约等于线锯的直径。

从图 4 可以看出, 随着摇摆运动的加入, 工件 与线锯的接触长度也随之发生变化; 这意味着摇摆 过程对接触长度会产生影响, 从而导致材料去除率 随之变化。本文以苏州赫瑞特公司 X07 M250×350$1 \mathrm{D}-\mathrm{O}$ 多线切割机为例进行分析(其运动方式与瑞士 梅耶博格公司的多线摇摆切割机的运动方式相似)。 为了便于分析, 考虑到线锯与工件的相对运动, 图 4 所示的工件摇摆也可以看作是线锯的摇摆, 如图 5 所示。线锯摇摆中心为切割晶棒的圆心点 $A$ 。在理论分 析中做如下假设: (1) 在切割过程中, 因线锯弯曲度较 小 ${ }^{[21]}$, 因此不考虑线锯弯曲对接触长度的影响, 假设 线锯与工件的接触为直线; (2) 不考虑锯切过程中, 线 锯的磨损对加工过程的影响; (3) 不考虑材料对加工过 程的影响, 仅对线锯的运动学进行分析; (4) 本文主要 研究一个摇摆周期中最大摇摆角度时的相互运动关 系，不对一个摇摆周期内的运动进行深入分析。

摇摆切割过程中线与晶圆的接触长度可以分两 个阶段进行分析, 第一个阶段为 $[-\Delta y, 0]$ 。此时切 割位置低于晶圆最低点的位置, 但是由于摇摆运动 的加入, 导致线锯与工件产生接触, 见图 $5 \mathrm{a}$ 所示。 在这个过程中接触长度为圆弧对应的弦长 $B C$, 即为

$$
B C=2 C D=2 \sqrt{A C^{2}-A D^{2}}
$$


第二个阶段为 $[0,2 r]$, 此时随着进给运动, 线 锯完成整个晶圆的切割加工, 见图 $5 \mathrm{~b}$ 所示。由于摇 摆运动的引入, 第二个阶段的接触长度并不等于圆 弧的弦长而是为部分弦长 $B C$, 即为

$$
B C=C D+D B=\sqrt{A C^{2}-A D^{2}}+A B \sin \theta
$$

两个阶段中摇摆切割过程不同位置处的最大接 触长度可表述为式(7)所示

$$
\left\{\begin{aligned}
l_{c r}(y)= & 2 \sqrt{r^{2}-[(r-y) \cos \theta]^{2}} \\
y & \in[-\Delta y, 0] \\
l_{\text {cr }}(y)= & \sqrt{r^{2}-[(r-y) \cos \theta]^{2}}+(r-y) \sin \theta \\
y & \in[0,2 r]
\end{aligned}\right.
$$

其中 $\Delta y$ 值可由式(8)求得

$$
\Delta y=\frac{(1-\cos \theta) r}{\cos \theta}
$$

式中, $\theta$ 为工件最大摇摆角度。

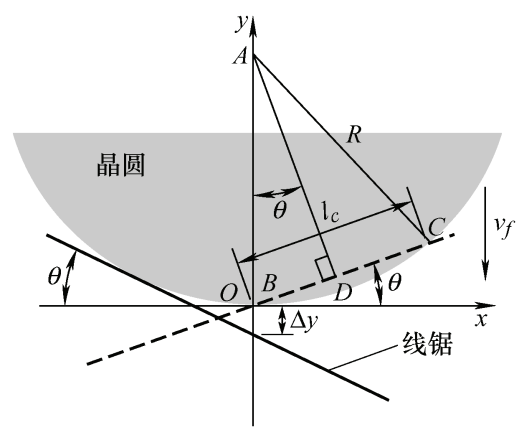

(a) 第一阶段接触长度

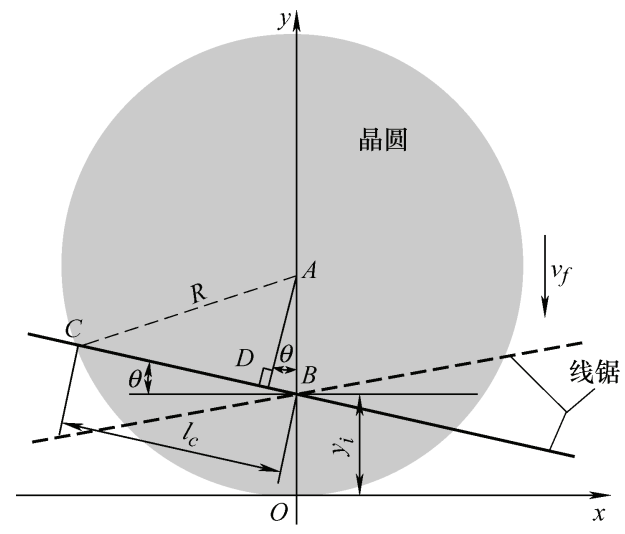

(b) 第二阶段接触长度

图 5 摇摆切割过程中接触长度示意图

式(3)、式(7)代入到式(4)可得在摇摆条件下的材 料去除率随切割位置 $y$ 变化的计算公式为式(9)所示

$$
Q(y)=l_{c r}(y) \cdot v_{f}(y) \cdot d
$$

由式(9)可以看出, 当加工工件尺寸固定时, 材 料去除率 $Q$ 是由工件的进给和摇摆运动而确定。

\section{2 加工运动参数对加工过程中材料去除率的影响}

本文仿真计算中选择切割工件半径 $r=50 \mathrm{~mm}$, 针
对线锯切割中的不同运动参数, 分别选择表 1 所示的 加工参数进行仿真计算, 表中标黑参数的为基础工艺 参数。将工艺参数代入到式(8)计算可以发现在实际加 工过程中, 第一个阶段仅占整个加工过程的 $0.7 \%$, 因 此在后续分析中, 主要考虑第二个阶段的影响。将各 工艺参数代入到式(9), 可以计算出不同工艺条件下的 材料去除率随晶圆切割过程的变化曲线如图 6 所示。

表 1 仿真工艺参数表

\begin{tabular}{lc}
\hline \multicolumn{1}{c}{ 工艺参数 } & 数值 \\
\hline 最大摇摆角度 $\theta /\left(^{\circ}\right)$ & $0,2,4,6,8,10$ \\
进给速比 $u /(\%)$ & $100,90,70,50,30$ \\
切割时间 $T / \mathrm{min}$ & $330,360,390,420,450$ \\
总耗线量 $M / \mathrm{m}$ & $3000 、 4000 、 5000 、 6000 、 7000$ \\
\hline
\end{tabular}

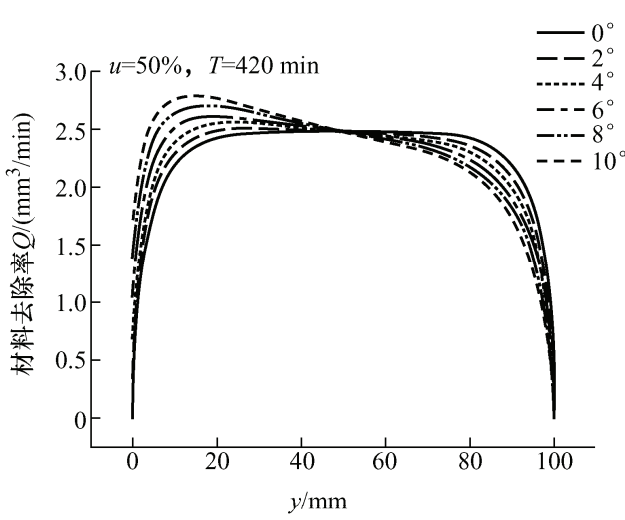

(a) 不同最大摇摆角度

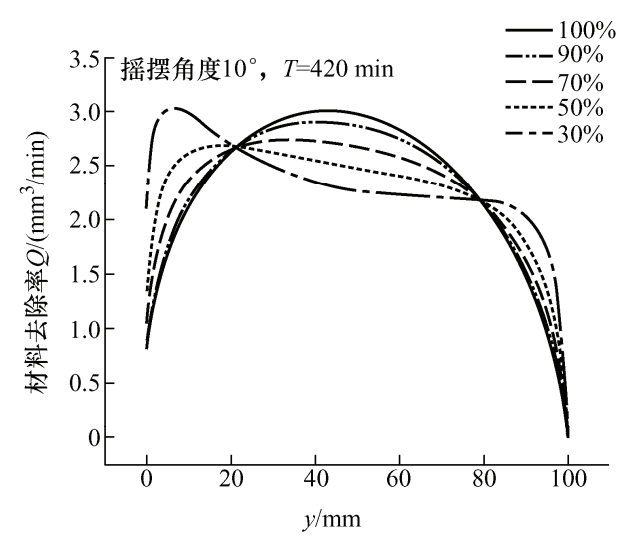

(b) 不同进给速比

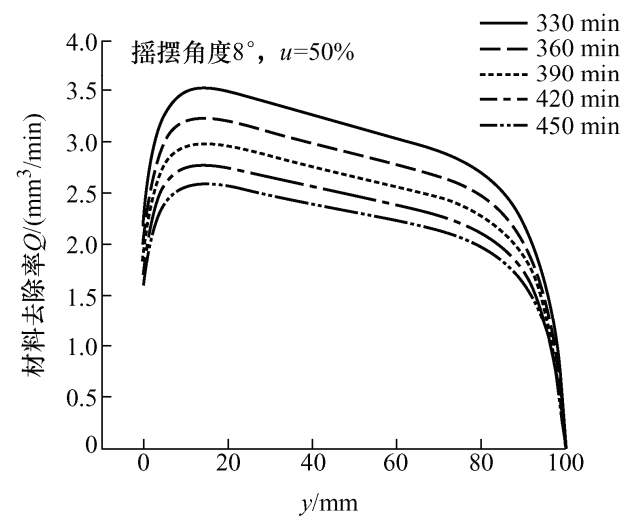

(c) 不同切割时间

图 6 不同工艺参数下的材料去除率的变化 
从图 6 中可以看出加工运动工艺参数的影响各 不相同。图 6a 表示不同摇摆角度下的材料去除率曲 线, 当摇摆角度为 $0^{\circ}$ 时为无摇摆切割, 从图 6 中 可以看出材料去除率前后端较为对称; 摇摆运动的 引入, 材料去除率的曲线形状随之发生变化。随着 摇摆角度的增大, 前端切割区域的材料去除率也随 之增大, 后端切割区域的材料去除率随之减小。

图 $6 \mathrm{~b}$ 表示了不同进给速比下材料去除率曲线, 当速比为 $100 \%$ 时为匀速进给切割, 即切割过程中进 给速度为定值, 从图中可以看出此时材料去除率曲 线为抛物线形状; 而随着进给速比的减小, 即两端 进给速度增加, 中间部分进给速度减小时, 材料去 除率也由中间突出向整体平稳转变, 但当进给速比 太小时, 反而会导致在两端的 $Q$ 明显高于中间部分 的 $Q$ (如图中速比为 $30 \%$ )。

图 6c 为不同切割总时间对材料去除率的影响, 当其它条件不变时, 延长切割总时间仅使得材料去 除率数值整体减小, 其形状没有明显变化。由于 $Q$ 仅 与工件的摇摆运动和进给运动相关, 与线锯的往复运 动无关, 因此总耗线量 $M$ 对材料去除率并无影响。

\section{3 多线摇摆往复切割的材料去除率模型的不足}

通过以上分析可以发现, 在切割过程中通过调 控工艺参数如进给速比、摇摆角度、切割时间等可 以实现对材料去除率的调控。对于实际生产而言, 希望能保证在整个切割过程中材料去除率保持稳 定, 即 $Q(y)$ 不随切割位置 $y$ 产生变化。从式(9)可知, 即要保证 $l_{c}(y)$ 与 $v_{f}(y)$ 乘积为常数, 见式(10)

$$
l_{c r}(y) \cdot v_{f r}(y)=k,(k \neq 0)
$$

则可得此时摇摆切割进给速度见式(11)

$$
\begin{gathered}
v_{f r}(y)=\frac{k}{l_{c r}(y)}= \\
\frac{k}{\sqrt{r^{2}-[(r-y) \cos \theta]^{2}}+(r-y) \sin \theta}
\end{gathered}
$$

当 $y=2 r$ 时, 此时分母为零, 则上述方程无解。 为保证方程可解, 在式(11)的分母项中加上非零常 数 $c$, 见式(12)

$$
Q(y)=\frac{d \cdot\left\{r^{2}\left[\frac{\arcsin (\cos \theta)}{\cos \theta}+2 \sin \theta+\frac{2 u}{1-u}\right]\right\} \cdot\left\{\sqrt{r^{2}-[(r-y) \cos \theta]^{2}}+(r-y) \sin \theta\right\}}{T\left[\sqrt{r^{2}-[(r-y) \cos \theta]^{2}}+(r-y) \sin \theta+\frac{u r}{1-u}\right]}
$$

但值得注意的是由于在式(12)的推导过程中引 入了常数 $c$, 从而导致此时等式(10)不成立, 即由于 常数的引入, 使得材料去除率 $Q$ 并不能在加工过程 中一直保持常数, 如式(20)所示

$$
v_{f r}(y) \cdot\left[l_{c r}(y)+c\right]=Q(y) \neq k
$$

$$
\begin{gathered}
v_{f r}(y)= \\
\frac{k}{\sqrt{r^{2}-[(r-y) \cos \theta]^{2}}+(r-y) \sin \theta+c}
\end{gathered}
$$

由式(12)可求出 $y=r$ 以及 $y=2 r$ 时的进给速度见 式(13)

$$
\left\{\begin{array}{l}
v_{f m}=k /(r+c),(y=r) \\
v_{f 0}=k / c,(y=2 r)
\end{array}\right.
$$

根据式(2)中对进给速比的定义，可以计算得到 $c$ 的值见式(14)所示

$$
c=\frac{u r}{1-u}
$$

从式(14)可知, 当 $u$ 确定时, 其 $c$ 值也随之可 以确定。

根据工件进给速度的分布, 可以推导出切割总 时间 $T$ 为

$$
T=\int_{0}^{2 r} \frac{1}{v_{f r}(y)} \mathrm{d} y
$$

将式(12)、(14)代入式(15)中可得

$$
T=\int_{0}^{2 r} \frac{\sqrt{r^{2}-[(r-y) \cos \theta]^{2}}+(r-y) \sin \theta+\frac{u r}{1-u}}{k} \mathrm{~d} y
$$

根据 $T$ 可求得 $k$ 值为

$$
k=\frac{r^{2}}{T}\left[\frac{\arcsin (\cos \theta)}{\cos \theta}+2 \sin \theta+\frac{2 u}{1-u}\right]
$$

将 $k$ 值及 $c$ 值代入到式(12)可到摇摆切割进给速 度在不同切割位置表达式为

$$
\begin{gathered}
v_{f r}(y)= \\
\frac{r^{2}\left[\frac{\arcsin (\cos \theta)}{\cos \theta}+2 \sin \theta+\frac{2 u}{1-u}\right]}{T\left[\sqrt{r^{2}-[(r-y) \cos \theta]^{2}}+(r-y) \sin \theta+\frac{u r}{1-u}\right]}
\end{gathered}
$$

此时摇摆切割过程中, 不同位置处的材料去除 率可由式(19)求得

从上述推导可以发现, 在切入和切出位置工 件与线锯的接触长度为 0 , 而实际生产过程中, 此 时的工件进给速度不可能无限大, 这是导致加工 过程中 $Q$ 难以保持常数的根本原因。这或许正是 目前实际生产中难以有效调控工艺参量的主要原 因之一。 


\section{3 固定单位长度新线材料去除体积 $R$ 的模型}

\section{1 固定新线材料去除体积的模型}

从上述分析可以看出, 仅从材料去除率的角度, 难以获得稳定的晶圆切割加工过程。同时从式(9)还 可以看出, 材料去除率的模型仅表征了工件的运动, 并没有涉及到工具的运动。而在磨削加工过程中, 相同材料去除率的条件下, 参与加工的磨粒数对加 工结果有着重要的影响 ${ }^{[23]}$ 。考虑到线锯表面磨粒分 布的较大不均匀性, 因此本文定义材料去除率 $Q$ 与 新线进给速度 $v_{w}$ 比值为单位长度新线材料去除体 积 $R$, 则可得到单位长度新线材料去除体积随切割 位置 $y$ 轴变化的计算公式如式(21)所示

$$
R(y)=\frac{Q(y)}{v_{w}(y)}
$$

磨削加工研究成果表明, 加工过程中, 保证稳 定的单颗磨粒材料去除体积将有利于保证加工过程 的平稳性以及加工工具的均匀磨损 ${ }^{[23]}$ 。因此, 本文 从保持加工过程中稳定的新线材料去除体积进行分 析, 见式(22)所示

$$
R(y)=\frac{l_{c r}(y) v_{f r}(y) d}{v_{w r}(y)}=E,(E \neq 0)
$$

由式(22)可求得摇摆切割过程中新线进给速度 $v_{w}(y)$ 见式(23)所示。

$$
v_{w r}(y)=\frac{l_{c r}(y) v_{f r}(y) d}{E}
$$

将式(23)代入式(1)化简后可得

$$
\begin{aligned}
& M=\int_{0}^{2 r} v_{w r}(y) \mathrm{d} y= \\
& \int_{0}^{2 r} \frac{l_{c r}(y) \cdot v_{f r}(y) \cdot d}{E} \cdot \frac{\mathrm{d} t}{v_{f r}(y)}= \\
& \int_{0}^{2 r} \frac{l_{c r}(y) \cdot d}{E} \mathrm{~d} t
\end{aligned}
$$

将式(7)代入式(24)化简得

$$
\int_{0}^{2 r} \frac{\left[\sqrt{r^{2}-[(r-y) \cos \theta]^{2}}+(r-y) \sin \theta\right] \cdot d}{E} \mathrm{~d} t
$$

由式(25)可求得 $E$ 值如式(26)所示

$$
E=\frac{\frac{2 d r^{2} \arcsin (\cos \theta)}{\cos \theta}+4 d \cdot \sin \theta r^{2}}{M}
$$

将式(7)、式(11)和式(26)代入到式(23), 可得新 线进给速度在不同切割位置的表达式见式(27)所示

$$
v_{w r}(y)=\frac{M \cdot\left\{r\left[\frac{\arcsin (\cos \theta)}{\cos \theta}+2 \sin \theta\right]+2 \frac{u r}{1-u}\right\} \cdot\left\{\sqrt{r^{2}-[(r-y) \cos \theta]^{2}}+(r-y) \sin \theta\right\}}{T \cdot\left[\sqrt{r^{2}-[(r-y) \cos \theta]^{2}}+(r-y) \sin \theta+\frac{u r}{1-u}\right] \cdot\left[\frac{2 r \arcsin (\cos \theta)}{\cos \theta}+4 \sin \theta r\right]}
$$

将式(19)和式(27)代入式(21), 可得单位长度新 线材料去除体积如式(28)所示

$$
\begin{aligned}
& R(y)=\frac{Q(y)}{v_{w r}(y)}= \\
& \frac{\frac{2 d r^{2} \arcsin (\cos \theta)}{\cos \theta}+4 d r^{2} \sin \theta}{M}
\end{aligned}
$$

比较式(19)和式(28)可以看出, 材料去除率的表 达式中含有位置变量 $y$, 而单位长度新线材料去除 体积 $R$ 只与切割过程中线锯直径 $d$ 、最大摇摆角度 $\theta$ 、切割晶圆半径 $r$ 以及总耗线量 $M$ 有关; 与切割 位置变量 $y$ 没有关系。当公式中的工艺参数预先设 定后, 切割过程中的 $R$ 即为一个固定值, 与切割位 置无关。这说明在加工过程中实现固定 $R$ 的去除加 工的思路是可行的。

\section{2 加工运动参数对新线材料去除体积的影响}

根据表 1 的工艺参数, 分别计算不同最大摇摆 角度 $\theta$ 以及不同耗线量 $M$ 条件下的单位长度新线材 料去除体积 $R$ 随加工过程的变化，如图 7 所示。从 图中可以看出, 正如文中所预期的, 通过合理调控
切割过程中工件进给速度以及新线进给速度随切割 位置的变化, 可以保证稳定 $R$ 的切割过程。

从图 7 还可以看出，随着最大摇摆角度的均匀 增加, $R$ 值随之均匀增加; 但随着耗线量的均匀增 加, $R$ 的值随之减小，但减小趋势逐渐放缓。进给 速比 $u$ 和切割总时间 $T$ 对 $R$ 并无影响。

\section{3 固定新线材料去除体积时的进给速度及材料 去除率的变化}

图 8 对比了在相同切割总时间 $T$ 下，三种不同 情况下的工件进给速度随加工过程的变化, 分别为 匀速切割; 无摇摆条件下，根据式(3)所计算的调整 后的工件进给速度；有摇摆条件下，固定 $R$ 的情况 下的所获得的工件进给速度, 由式(18)得到。其它 工艺条件如下：摇摆角度 $8^{\circ}$, 进给速比 $50 \%$, 切割 时间 $420 \mathrm{~min}$ 。

从图 8 中可以看出, 与均匀进给速度(直线)相 比, 调整后的工件进给速度在切割工件两端时, 其 进给速度明显提高, 但在切割工件中段时, 进给速 度明显减慢; 而与无摇摆条件下的进给速度(短划线) 
相比, 虽然两者有着相似的变化曲线, 但仍然会有 细微的差别; 无摇摆条件下, 进给速度呈现对称分 布的, 但加入摇摆后进给速度不再为对称分布, 这 与摇摆运动导致线锯提前与工件接触有关。

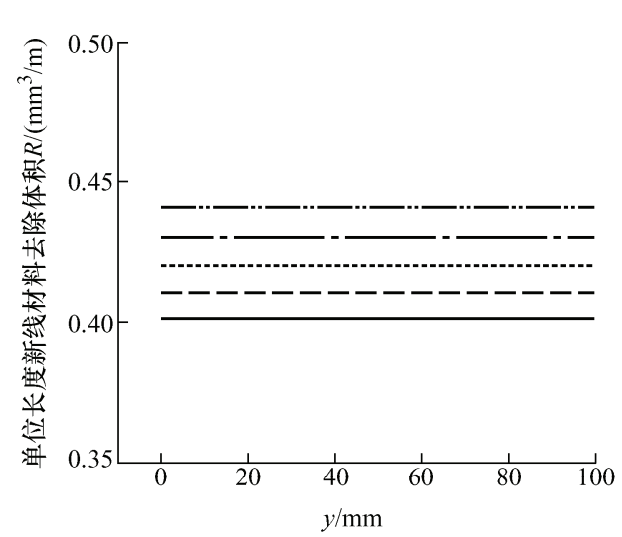

(a) 不同最大摇摆角度

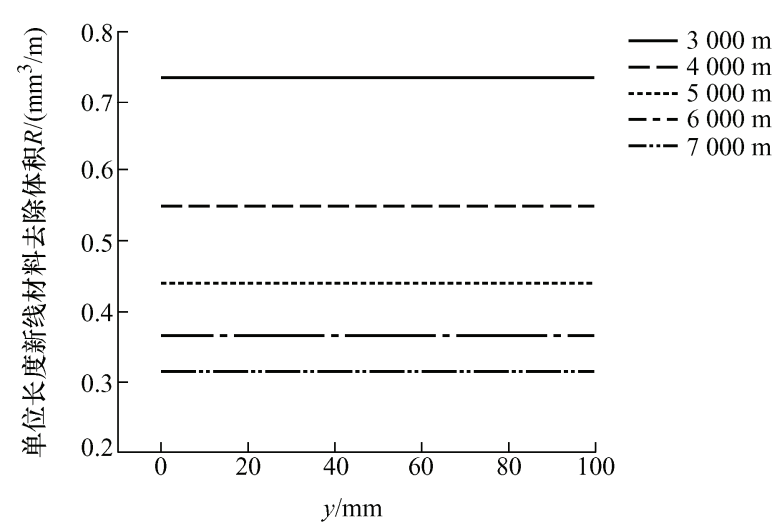

(b) 不同耗线量

图 7 不同工艺参数下 $R$ 的变化

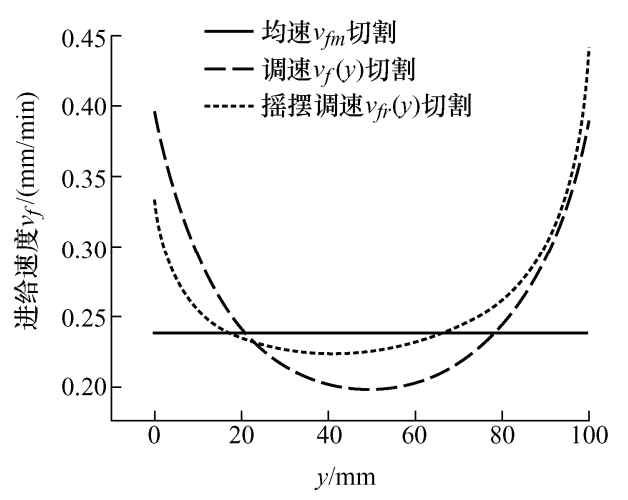

图 8 不同切割情况进给速度

不同条件下的材料去除率如图 9 所示。其工艺 条件如下: 摇摆角度 $8^{\circ}$, 进给速比 $50 \%$, 切割时间 $420 \mathrm{~min}$ 。图中直线和点线表示切割过程中, 均匀进 给速度条件有摇摆与无摇摆条件下 $Q$ 的变化; 短划 线的曲线为不考虑 $R$ 固定条件下，有无摇摆条件下 的 $Q$ 的变化(也是目前生产中常用的工艺参数设定 方法); 而双点划线的曲线是在考虑了固定 $R$ 去除加
工条件下, 修正了进给速度后得到的 $Q$ 的变化。总 体比较可以发现, 无论那种条件下, 加工过程中的 $Q$ 都无法完全保持稳定。随着摇摆运动的加入, 加 工过程中的最大 $Q$ 值相比于无摇摆条件都显著减 小, 均匀进给速度条件下, 摇摆运动的加入可以有 效平缓加工过程中 $Q$ 的变化, 修整后的 $Q$ 与修整前 的 $Q$ 在形状上大致相似, 但在局部上有所不同, 其 变化介于均匀进给速度和修整前的进给速度之间。

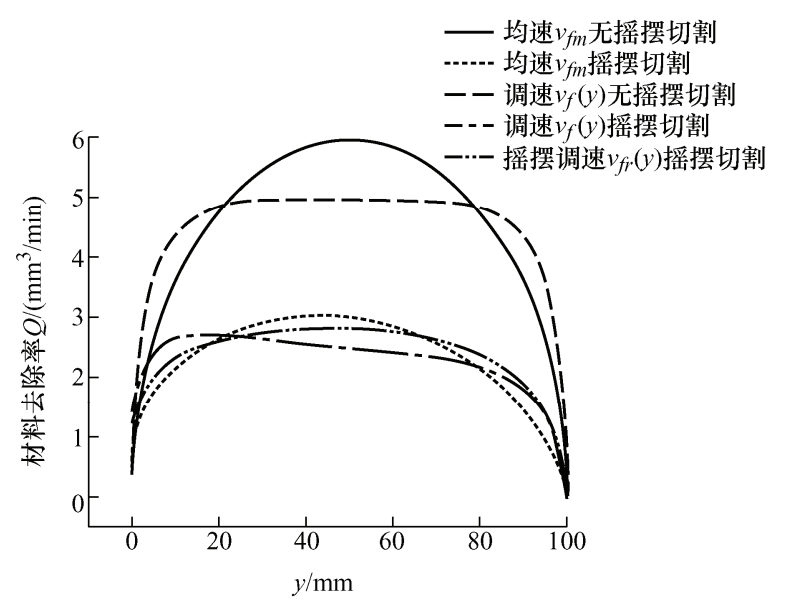

图 9 不同切割情况材料去除率

\section{4 切割试验}

切割试验在苏州赫瑞特公司 X07M250×350-1D -O 多线切割机上进行, 切割线锯采用日本住友电镀金 刚石线锯, 线径为 $0.18 \mathrm{~mm}$ 、磨粒大小 $30 \sim 40 \mu \mathrm{m}$; 切割工件为直径 $100 \mathrm{~mm}$ 的 $4 H-N$ 型单晶碳化硅, 晶棒长度 $20 \mathrm{~mm}$; 最大摇摆角度为 $10^{\circ}$, 摇摆周期 为 $4 \mathrm{~s}$, 张紧力为 $45 \mathrm{~N}$, 最大线速度为 $25 \mathrm{~m} / \mathrm{s}$, 往 复周期: $86 \mathrm{~s}$, 进给速比为 $50 \%$, 单片耗线量采用 $327 \mathrm{~m} / \mathrm{pcs}$, 总切割时间为 $340 \mathrm{~min}$ 。进给速度和新 线进给速度曲线分别采用企业提供(工艺 A) 和本文 修整的工艺参数(工艺 B) 进行切割, 其随加工位置的 变化曲线如图 10 和图 11 所示。两种工艺的 $Q$ 和 $R$ 曲线如图 12 和图 13 所示。采用 Tropel MSP150 平 面度仪对加工后的晶圆面型精度进行测量, 主要包 括: 厚度总偏差(TTV), 翅曲度(WARP), 弯曲度 (BOW)。

两种不同工艺所加工得到的晶圆质量结果见图 14 所示。从图 14 中可以看出, 两种工艺加工后的 TTV 值没有明显差别, 这是因为所加工用的机床和 工具都相同，因此，晶片的厚度大致相同；但是反 映晶片形状的尧曲度和弯曲度有着明显的差别。对 于采用固定 $R$ 的加工工艺所加工的晶片, 其趐曲度 和弯曲度都有明显地减小。图 15 是两种工艺条件下 
切割出来的晶片的典型形貌, 从形貌图中也可以看 出, 采用工厂现有工艺参数所加工的晶片在其中部 产生了明显的凸起; 相比而言, 采用固定 $R$ 而调整 后的工艺参数所加工的晶片其表面更加平稳, 没有 观察到明显的凹凸。

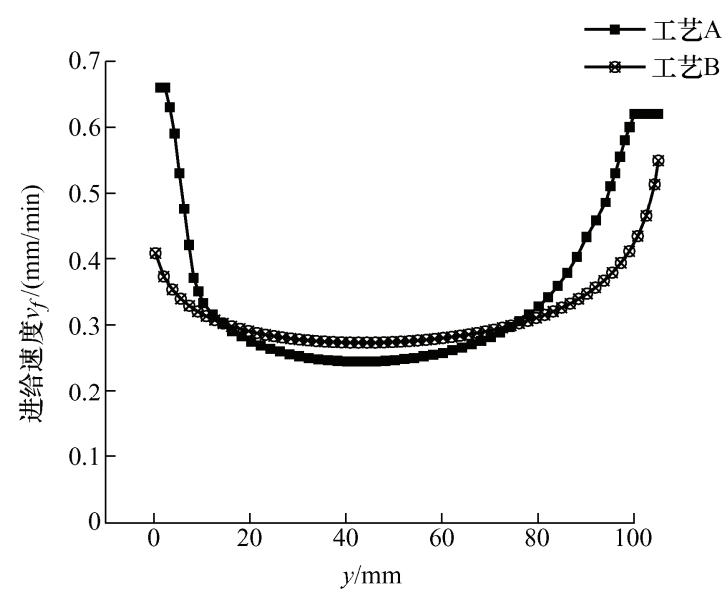

图 10 不同工艺进给速度曲线

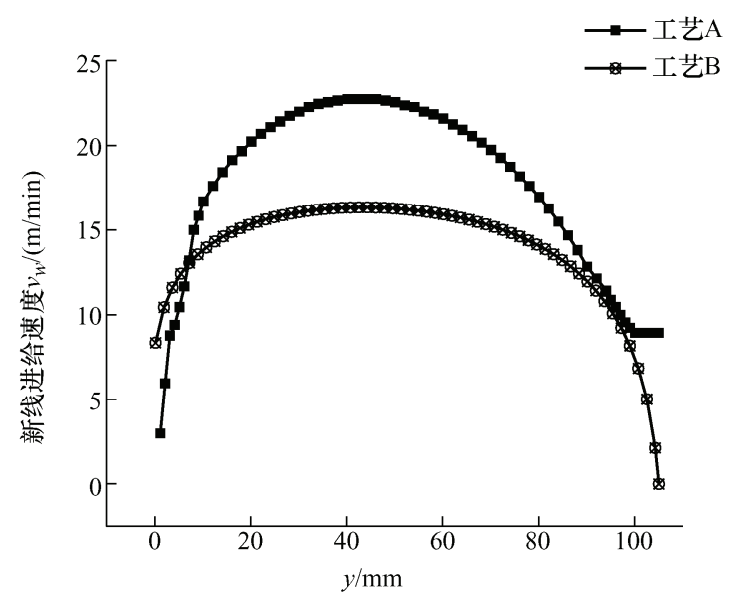

图 11 不同工艺新线进给速度曲线

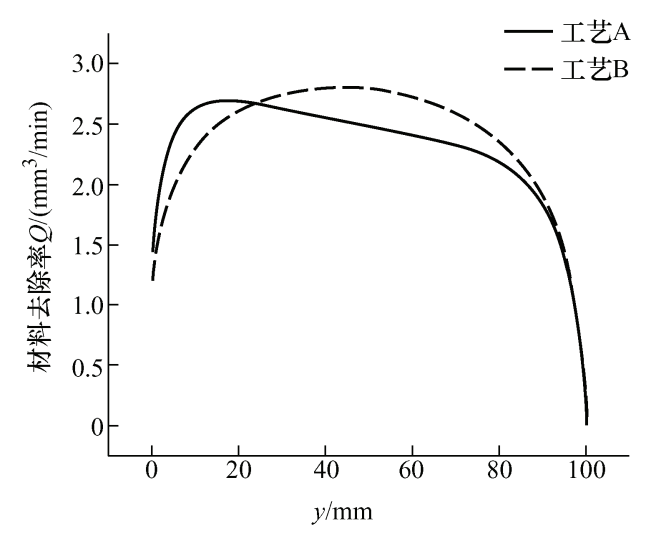

图 12 材料去除率 $Q$ 的变化曲线

从加工过程中各参数的变化曲线(图 10〜13), 可以发现, 虽然工艺 $\mathrm{A}$ 采用改变加工过程中工件进 给速度的方式来调整材料去除率, 使得加工过程中
的材料去除率变得平稳(图 12), 但是其新线进给速 度没有做相应的调整(图 11), 因此导致其新线材料 去除体积在加工过程中有明显波动(图 13)。在加工 前段, 其新线材料去除体积明显高出, 而加工后段 也存在新线材料去除体积的变化。而从加工结果也 可以看了, 其加工后的表面在工件两端也出现了较 大的波动(图 15a)。相比而言, 文中基于固定 $R$ 的切 割思路, 对工件进给速度以及新线进给速度进行相 应的调整。从材料去除率的变化曲线而言, 其随加 工过程的变化并不均匀(图 12), 但是其新线材料去 除体积在加工过程中可以保证稳定(图 13)。其最终 加工结果发现工件的表面更为平稳。试验结果表明, 采用固定 $R$ 的加工工艺方法可以有效地提高多线摇 摆往复式切割晶片的加工质量。

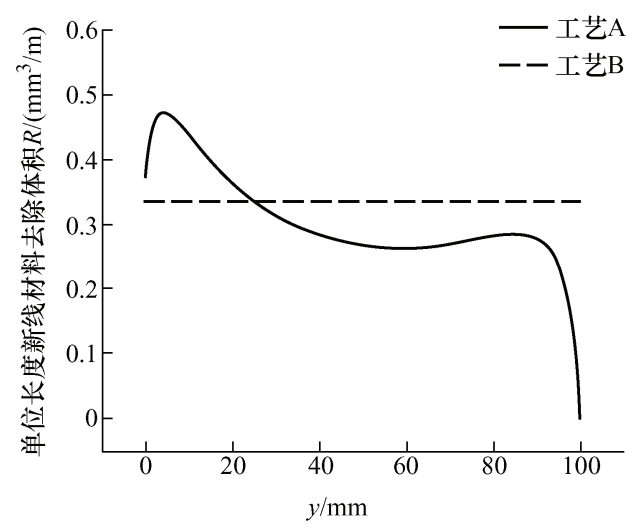

图 13 单位长度新线材料去除体积 $R$ 的变化曲线

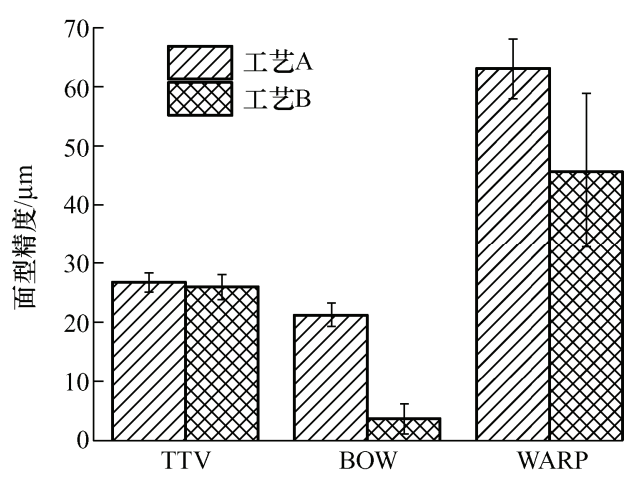

图 14 不同工艺加工的晶圆切割质量

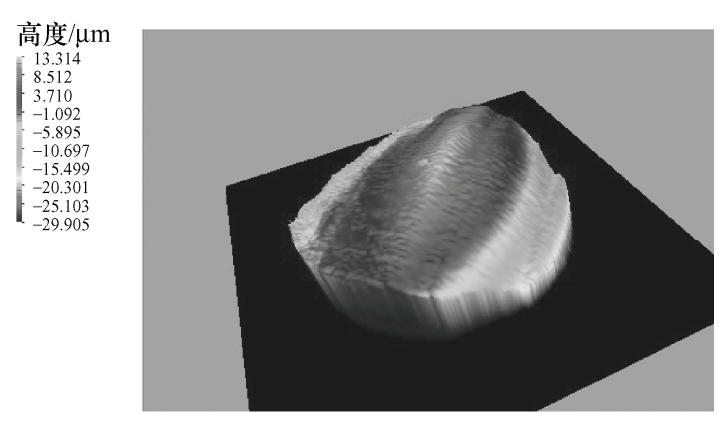

(a) 工艺条件A 


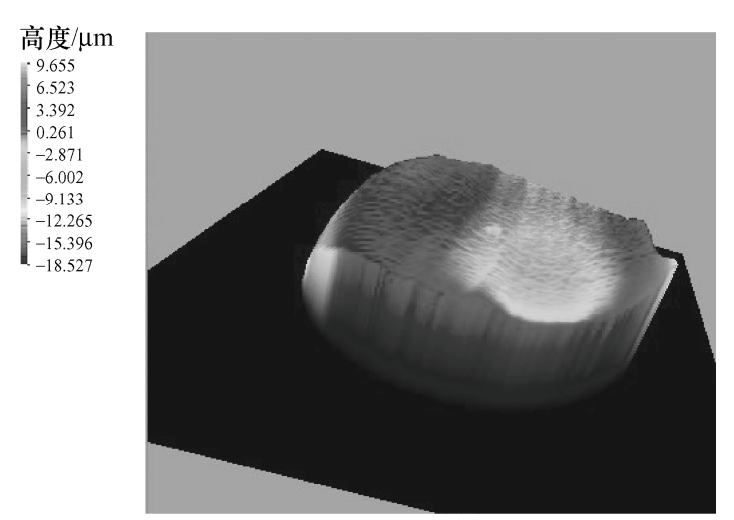

(b) 工艺条件B

图 15 不同工艺条件下的切割片高度云图

\section{5 结论}

(1) 针对多线摇摆往复式线锯切割加工这种新 的加工方法, 理论分析了工件进给运动、工件摇摆 运动及线锯往复运动三个主要的运动方式; 建立了 其加工过程中的材料去除率 $Q$ 的理论模型, 并理论 分析了不同加工工艺参数对 $Q$ 的影响规律, 结果表 明摇摆角度的增加会导致切割过程中, 前端切割区 域的 $Q$ 随之增加, 而后端切割区域的 $Q$ 随之减小。 随着进给速比的减小, 材料去除率由中间突出向整体 平稳转变, 但过小进给速比会导致两端的材料去除率 过大; 切割总时间的增加使得 $Q$ 随之减小, 但形状 没有变化。分析结果同样表明, 由于受到晶圆形状的 影响, 加工过程中材料去除率难以完全保持稳定。

(2) 提出了单位长度新线材料去除体积的理论 模型, 理论推导表明加工过程中 $R$ 仅与预设工艺参 数有关, 而与线锯切割位置无关。基于此模型, 提 出了固定单位长度新线材料去除体积的优化加工工 艺, 并推导出了固定 $R$ 时工件进给速度及新线进给 速度的理论公式。理论分析结果表明随着最大摇摆 角度的增加, $R$ 值随之均匀增加; 随着耗线量的增 加, $R$ 的值随之减小, 但减小趋势是逐渐放缓。

(3) 采用工厂切割工艺和本文所提出的固定 $R$ 的加工工艺进行切割对比研究, 切割试验结果表明优 化后的工艺参数可以有效地提高晶片的加工质量。

\section{参 考 文 献}

[1] 贺东葛, 王家鹏, 刘国敬. 碳化硅半导体材料应用及发 展前景[J]. 电子工业专用设备, 2018(3)：1-3.

HE Dongge, WANG Jiapeng, LIU Guojing, et al. Application and development prospect of silicon carbide semiconductor materials[J]. Equipment for Electronic Products Manufacturing, 2018(3): 1-3.
[2] 魏昕, 杜宏伟, 袁慧, 等. 晶片材料的超精密加工技术 现状 [J]. 组合机床与自动化加工技术, 2004(3): 75-79. WEI Xin, DU Hongwei, YUAN Hui, et al. Ultra-precision machining technology for crystal wafers[J]. Combined Machine Tool and Automatic Machining Technology, 2004(3): 75-79.

[3] 孟剑峰. 环形电镀金刚石线锯加工技术及加工质量研 究[D]. 济南: 山东大学, 2006.

MENG Jianfeng. Research on machined technology and machined quality of endless electroplated diamond wire saw[D]. Jinan: Shandong University, 2006.

[4] WANG X Y, LI Y, LI S J . Study on the impact of the cutting process of wire saw on $\mathrm{SiC}$ wafers[J]. Applied Mechanics and Materials, 2011，120: 593-597.

[5] HUANG H, ZHANG Yuxing, XU Xipeng. Experimental investigation on the machining characteristics of singlecrystal $\mathrm{SiC}$ sawing with the fixed diamond wire[J]. International Journal of Advanced Manufacturing Technology, 2015, 81(5-8): 955-965.

[6] HARDIN C W, QU J, SHIH A J. Fixed abrasive diamond wire saw slicing of single crystal $\mathrm{SiC}$ wafers[C]//ASME International Mechanical Engineering Congress \& Exposition. 2003.

[7] ZHANG M J. Experimental study on slicing of sapphire with fixed abrasive diamond wire saw[J]. International Journal of Mechanical, Aerospace, Industrial, Manufacturing Engineering, 2015, 9(5): 661-664.

[8] GAO Y, GE P, LIU T . Experiment study on electroplated diamond wire saw slicing single-crystal silicon[J]. Materials Science in Semiconductor Processing, 2016, 56: 106-114.

[9] 高玉飞, 陈阳, 葛培琪. $\mathrm{SiC}$ 单晶线锯切片微裂纹损伤 深度的有限元分析[J]. 西安交通大学学报, 2016(12): y1-y6.

GAO Yufei, CHEN Yang, GE Peiqi. Finite element analysis on wafer micro-crack damage depth of $\mathrm{SiC}$ crystal in wire saw slicing process[J]. Journal of Xi'an Jiaotong University, 2016(12): y1-y6.

[10] HUANG X, HUANG H, GUO H . Simulation and experimental research on the slicing temperature of the sapphire with diamond wire[J]. International Journal of Computational Methods, 2018: 1843003.

[11] 张辽远. 电镀金刚石线锯超声波切割试验装置的研制 和加工机理的研究[D]. 长春: 长春理工大学, 2007.

ZHANG Liaoyuan. Research of processing principle and development of experiment setup for electroplated diamond 
wire saw with ultrasonic vibration[D]. Changchun: Changchun University of Science and Technology, 2007.

[12] MÖLLER H J. Basic mechanisms and models of multi wire sawing[J]. Advanced Engineering Materials, 2010, 6(7): 501-513.

[13] LIEDKE T, KUNA M . A macroscopic mechanical model of the wire sawing process[J]. International Journal of Machine Tools \& Manufacture, 2011, 51(9): 711-720.

[14] KIM H, KIM D, KIM C, et al. Multi-wire sawing of sapphire crystals with reciprocating motion of electroplated diamond wires[J]. CIRP Annals- Manufacturing Technology, 2013, 62(1): 335-338.

[15] KIM D, KIM H, LEE S, et al. Effect of initial deflection of diamond wire on thickness variation of sapphire wafer in multi-wire saw $[\mathrm{J}]$. International Journal of Precision Engineering and Manufacturing-Green Technology, 2015, 2(2): 117-121.

[16] BHAGAVAT S, KAO I . A finite element analysis of temperature variation in silicon wafers during wiresaw slicing $[\mathrm{J}]$. International Journal of Machine Tools and Manufacture, 2008, 48(1): 95-106.

[17] SHINYA M, YAZAWA T, OTUBO T. Study on the slicing of sapphire using a wire tool[J]. Advanced Materials Research, 2015, 806: 333-337.

[18] ABE Y, ISHIKAWA K I, SUWABE H . Effects of thermal deformation of multi-wire saw's wire guides and ingot on slicing accuracy[J]. Key Engineering Materials, 2009, 389-390: 442-447.
[19] MAEDA $\mathrm{H}$, TAKANABE $\mathrm{R}$, TAKEDA $\mathrm{A}$, et al. High-speed slicing of $\mathrm{SiC}$ ingot by high-speed multi wire saw[C]//Trans Tech Publications, 2014: 771-775.

[20] 林志树, 黄辉, 郑生龙. 多线往复式线锯切割中单位长 度材料去除量的理论分析与试验研究 [J]. 机械工程学 报，2018，54(13): 208-214.

LIN Zhishu, HUANG Hui, ZHENG Shenglong. Theoretical analysis and experimental study on material removal per length of wire in the cutting of multi-wire saw with reciprocating $[\mathrm{J}]$. Journal of Mechanical Engineering, 2018, 54(13): 208-214.

[21] CLARK W I, SHIH A J, HARDIN C W, et al. Fixed abrasive diamond wire machining-part i : Process monitoring and wire tension force $[\mathrm{J}]$. International Journal of Machine Tools \& Manufacture, 2003, 43(5): 523-532.

[22] TEOMETE E. Investigation of long waviness induced by the wire saw process[J]. Proceedings of the Institution of Mechanical Engineers, Part B: Journal of Engineering Manufacture, 2011, 225(7): 1153-1162.

[23] MALKIN S, GUO C. Grinding technology: Theory and applications of machining with abrasives[J]. International Journal of Machine Tools \& Manufacture, 1991, 31(3): 435-436.

作者简介: 杨沈, 女, 1995 年出生。主要研究方向为脆性材料精密加工。 E-mail: yangqin199@qq.com 黄辉(通信作者), 男, 1974 年出生, 博士, 教授, 博士研究生导师。主 要研究方向为脆性材料精密加工。

E-mail: huangh@hqu.edu.cn 